\title{
GALACTIC-CENTER JET OF 4-kpc LENGTH
}

\author{
Y. SOFUE ${ }^{1}$, W. REICH ${ }^{2}$, and P. REICH ${ }^{2}$ \\ 1.Institute of Astronomy, Univ. of Tokyo, Mitaka, Tokyo 181, Japan \\ 2.MPIfR, Auf dem Hügel 69, D-5300 Bonn 1, FRG
}

\begin{abstract}
We report the detection of a 4-kpc long, highly collimated radio feature emanating from the galactic center. This feature, which may be cylindrical in shape, is some $200 \mathrm{pc}$ in diameter and extends almost perpendicular to the galactic plane. The structure may possibly be the remnant of a one-sided or highly asymmetric jet from the nucleus, or it might be a magnetic tornado produced by a twisted poloidal magnetic field between the disk and halo.
\end{abstract}

\section{INTRODUCTION}

Edge-on spiral galaxies often exhibit large-scale ejection structures of a fewkiloparsec scale (e.g. Duric et al 1983), while it is not clear if our own Galaxy has experienced such an energetic jet ejection from the nucleus. This simple question, "Has the Milky Way a kiloparsec scale jet?", might be answered by a detailed analysis of existing radio data like the all-sky survey at $408 \mathrm{MHz}$ (Haslam et al 1982). In this paper we examine the $408 \mathrm{MHz}$ data in detail by extensively applying the background-filtering (BGF) technique with which we can enhance fine structures such as jet-like features and spurs by filtering out the galactic disk emission. In addition we made new observations at $1408 \mathrm{MHz}$ using the Effelsberg 100-m telescope. Based on these data we report the possible detection of a 4-kiloparsec-long jet emanating from the galactic nucleus, which we call the Galactic Center Jet (GCJ) (Sofue et al 1988).

\section{THE GALACTIC-CENTER JET - Appearance and Location -}

Fig. 1 shows a BGF map of the central $24^{\circ} \times 30^{\circ}$ of the galactic center at 408 $\mathrm{MHz}$ at a resolution of $50^{\prime}$. In the figure we notice a prominent spur emanating from the galactic center region toward high latitudes almost vertically, reaching as high as $b \sim 25^{\circ}$. This spur was already noticed by Haslam et al (1981), but they thought it to be a local object. The figure shows that the feature is composed of a straight (or slightly waving) ridge which bends at $b \simeq 9^{\circ}$ and $b \simeq 17^{\circ}$. The lower 


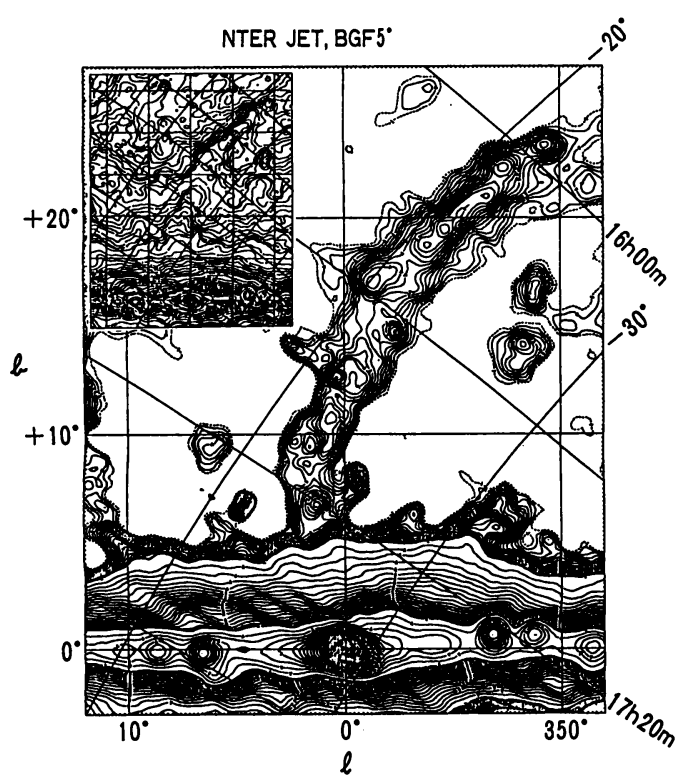

Fig.1: $408 \mathrm{MHz}$ map of the central $24^{\circ} \times 30^{\circ}$ of the Galaxy, where the background smooth structures of scale sizes greater than $5^{\circ}$ have been subtracted. Original data, as inserted in the upperleft corner, were taken from Haslam et al (1982). The Galactic Center Jet (GCJ) is the prominent ridge emanating from the galactic center perpendicular to the disk. The angular resolution is $50^{\prime}$. Contour interval near the GCJ is $1 \mathrm{~K} T_{\mathrm{b}}$.

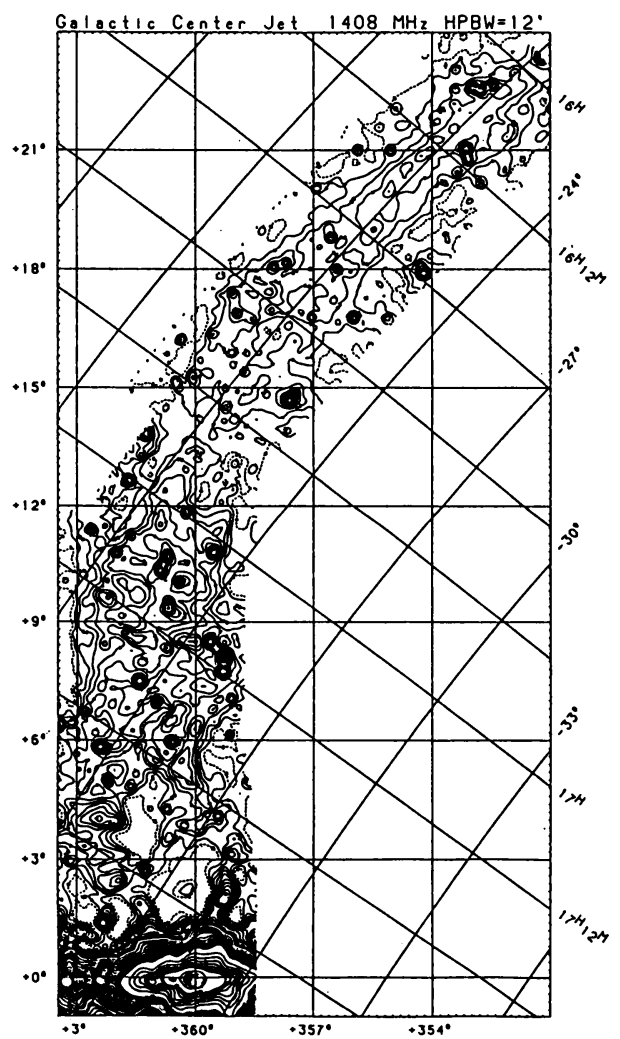

Fig.2: $1408 \mathrm{MHz}$ maps of the GCJ taken with the 100-m telescope at a resolution of 9'.4. The contour interval is $0.3 \mathrm{~K} T_{\mathrm{b}}$. Note the connection of the structure to the galactic center lobe (GCL) at $l=-0^{\circ} .6, b=1^{\circ}$, and to the galactic center. 
part seems to be emanating just from the galactic center, although the connection to the nucleus below $b \simeq 5^{\circ}$ is not clear from the $408 \mathrm{MHz}$ data because of the strong contamination of the galactic disk emission. Moreover the spur seems to be double-ridged at $b \simeq 9^{\circ} \sim 17^{\circ}$, which suggests a cylinder shape of the feature. Finally we note that the feature is one-sided, and no clear southern extension is visible.

In order to examine the connection of the feature to the galactic center we made $1408 \mathrm{MHz}$ continuum observations using the Effelsberg 100-m telescope at a resolution of $9^{\prime} .4$. The result is shown in Fig. 2, where we can see that the lower part $\left(b<5^{\circ}\right)$ of the spur is clearly connected to the galactic center by a thin ridge running from $(l, b) \sim\left(-0^{\circ} .6,0^{\circ} .8\right)$ to $\sim\left(0^{\circ} .5,6^{\circ}\right)$. The lower end of this ridge is also connected to the Galactic Center Lobe (Sofue and Handa 1984). From these facts we may conclude that the present feature is a structure physically connected to the galactic center. From the appearence and, in particular, from the connection to the galactic center, we may suppose that this object is a structure related to the galactic-center activity, and we call this object "the Galactic Center Jet"(GCJ).

\section{SCALES AND ENERGETICS}

If the GCJ is located near the galactic center of distance $8 \mathrm{kpc}$, the height, $b \sim 25^{\circ}$, is about $3.6 \mathrm{kpc}$ and the length along the jet is approximately $4 \mathrm{kpc}$. In contrast its width is as narrow as $200 \mathrm{pc}$, and therefore the jet is a highly collimated object.

The total flux density of the whole structure at $408 \mathrm{MHz}$ is $S \sim 10^{3} \mathrm{Jy}$. The spectral index between 408 and $1408 \mathrm{MHz}$ is roughly $\alpha \sim-0.6$ with $S \propto \nu^{\alpha}$. This indicates that the GCJ is a nonthermal object composed of magnetic fields and cosmic rays. The radio luminosity integrated from 0.1 to $100 \mathrm{GHz}$ is then $L_{\mathrm{R}} \sim 10^{36} \mathrm{erg} \mathrm{s}^{-1}$.

If we assume an equipartition between magnetic and cosmic-ray energies, we obtain a rough idea about the magnetic field strength: $B>$ a few $\mu \mathrm{G}$. Magnetic energy involved in the structure may be therefore $E_{\mathrm{mag}}>$ a few $10^{51} \mathrm{erg} \mathrm{s} \mathrm{s}^{-1}$. Then the radio decay time of the structure is $\tau_{\text {r.d. }} \sim E_{\mathrm{mag}} / L_{\mathbf{R}} \sim>10^{7 \sim 8}$ y. If the magnetic diffusion or an expansion due to the magnetic pressure is dominant, we have another time scale, which is approximately estimated as $\tau_{\text {m.d. }} \sim d / 2 V_{\mathbf{A}} \sim$ some $10^{6} \mathrm{y}$. Here $d$ is the diameter $(\sim 200 \mathrm{pc})$ and $V_{\mathrm{A}} \sim B(4 \pi \rho)^{-1 / 2} \sim 10^{2} \mathrm{~km}$ $\mathrm{s}^{-1}$ is the Alfven velocity near the jet with $\rho \sim 10^{-3 \sim-4} m_{\mathrm{H}} \mathrm{cm}^{-3}$ being the gas density in the halo.

\section{ORIGIN OF GCJ}

From its appreance and connection to the galactic center we may conclude that the structure detected here is likely a jet or a remnant of a jet ejected from the galactic center. It is not likely that the structure is a local object like a part of SNR 
because of its straight and cylindrical nature. The one-sidedness, or the fact that the structure stops suddenly near the galactic center and no southern extension is visible, may also be taken as the evidence for its physical connection to the galactic center. This may also exclude the possibility of a background extragalactic jet.

As to the acceleration mechanism we may envision the following models:

(A) A jet ejected from the galactic center or the remnant of a relativistic plasma beam from the nucleus: If it is still relativistic, the one-sidedness may be explained by a tilt of the jet toward us in the northern half, while away from us in the south of the galactic plane. However, from the low brightness of the jet it seems not likely that the jet is still highly relativistic. In this sense it may be either a remnant of a true one-sided jet which was ejected more than $\sim 10^{6} \mathrm{y}$ ago, or a part of an asymmetric double-sided jet. In this context it should be mentioned that the "low-energy jet" emanating from Sgr A toward the south as found by Yusef-Zadeh et al (1987) might be a counterpart to the GCJ, although the scales are very different from each other.

(B) A magnetic tornado: It is known that the magnetic field in the central region of our Galaxy is predominantly poloidal (e.g. Sofue 1988, in this issue). A large-scale poloidal field may be twisted by the differential rotation between the disk and halo gases. The twist will then strengthen the field and accelerate plasma as well cosmic rays from the disk toward the halo along the field lines. This is a similar mechanism to the magnetic twist acceleration mechanism as discussed by Shibata (1988, in this issue).

In either case, or in other possible cases, more convincing modeling of this prominent jet structure in our Milky Way center is highly desired. In particular, the relationship of the jet with the nuclear disk, which may be most extensively studied compared to those in external galaxies, will give a substantial clue to understand the acceleration mechanism of a galaxy-scale jet.

\section{References}

Duric,N., Sandquist,E.R., Crane,P.C., Bignell,R.C., Davis,L.E., 1983, Ap.J. Let., 273, L11

Haslam,C.G.T., Salter,C.J., Stoffel,H., Wilson,W.E., 1982, A. Ap. Suppl. 47, 1 Haslam,C.G.T., Klein,U., Salter,C.J., Stoffel,H., Wilson,W.E., Cleary,M.N., Cooke, D.J., Thomasson,P. 1981, A. Ap., 100, 209

Shibata, K. 1988, in this issue

Sofue, Y. 1988, in this issue.

Sofue,Y., Handa,T. 1984, Nature, 310, 568

Sofue,Y., Reich,W., Reich.P., 1988, Preprint

Yusef-Zadeh,F., Morris,M., Slee,O.B., Nelson,G,J. 1987, Ap.J., 300, L47 